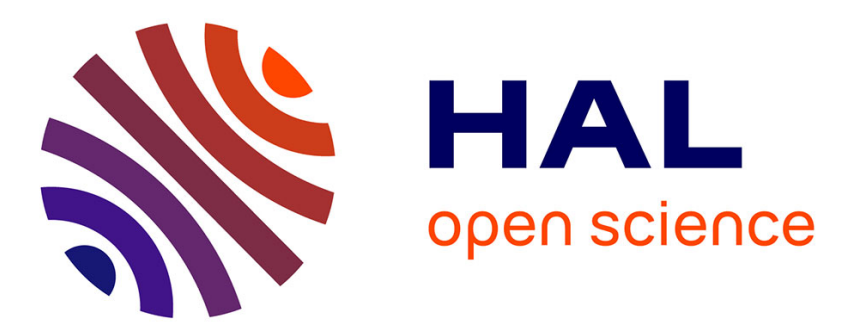

\title{
Sur l'utilisation de la force de Skyrme dans l'étude des systèmes nucléaires en condensation pionique G. Do Dang, A.A. Raduta
}

\section{To cite this version:}

G. Do Dang, A.A. Raduta. Sur l'utilisation de la force de Skyrme dans l'étude des systèmes nucléaires en condensation pionique. Journal de Physique Lettres, 1980, 41 (24), pp.585-587. 10.1051/jphyslet:019800041024058500 . jpa-00231852

HAL Id: jpa-00231852

https://hal.science/jpa-00231852

Submitted on 1 Jan 1980

HAL is a multi-disciplinary open access archive for the deposit and dissemination of scientific research documents, whether they are published or not. The documents may come from teaching and research institutions in France or abroad, or from public or private research centers.
L'archive ouverte pluridisciplinaire HAL, est destinée au dépôt et à la diffusion de documents scientifiques de niveau recherche, publiés ou non, émanant des établissements d'enseignement et de recherche français ou étrangers, des laboratoires publics ou privés. 


\title{
Sur l'utilisation de la force de Skyrme dans l'étude des systèmes nucléaires en condensation pionique
}

\author{
G. Do Dang \\ Laboratoire de Physique Théorique et Hautes Energies (*), Université de Paris-Sud, 91405 Orsay, France
}

\author{
et A. A. Raduta \\ Institute of Physics and Nuclear Engineering, P.O. Box 5206, Bucarest, Roumanie
}

(Reçule 18 septembre 1980, accepté le 23 octobre 1980)

Résumé. - Il est montré que la force de Skyrme n'est pas adaptée pour l'étude des systèmes nucléaires en condensation pionique où les nucléons possèdent une structure en spin-isospin particulière. Abstract. - It is shown that the Skyrme force is not adapted for the study of pion-condensed nuclear systems where
the nucleons have a particular spin-isospin structure.

La force de Skyrme [1] de portée nulle et dépendante de la densité a été largement employée dans l'étude des systèmes nucléaires, souvent avec des succès remarquables [2]. C'est une force phénoménologique dont les paramètres sont définis pour reproduire les propriétés connues des noyaux atomiques. Etant phénoménologique, elle est bien entendue supposée n'être adaptée qu'à l'étude des systèmes pour lesquels elle a été fabriquée, c'est-à-dire les systèmes dont la densité est voisine de la densité nucléaire et possédant des propriétés d'invariance usuelles (spécialement l'invariance par le renversement du temps). Toutefois, étant donné l'attrait dû à sa simplicité d'utilisation, il serait intéressant de savoir si cette force pourrait être utilisée pour des systèmes ayant des structures particulières comme par exemple la structure laminée des nucléons en condensation pionique. Nous allons montrer que, malheureusement, ce n'est pas le cas et que son utilisation dans ces conditions pourrait conduire à de sérieuses difficultés.

Sous sa forme usuelle, la force de Skyrme s'écrit

où

$$
V_{\mathrm{s}}=V_{\mathrm{c}}+V_{\mathrm{s} 0}+V_{3}
$$

$$
\begin{aligned}
& V_{\mathrm{c}}=t_{0}\left(1+x_{0} P_{\sigma}\right) \delta_{12}+\frac{t_{1}}{2}\left(\mathbf{k}^{\prime 2} \delta_{12}+\delta_{12} \mathbf{k}^{2}\right)+ \\
& +t_{2}\left(\mathbf{k}^{\prime} . \delta_{12} \mathbf{k}\right) \\
& V_{\mathrm{s} 0}=i W\left(\sigma_{1}+\sigma_{2}\right) .\left(\mathbf{k}^{\prime} \wedge \delta_{12} \mathbf{k}\right) \\
& V_{3}=t_{3} \delta_{12} \delta_{23} .
\end{aligned}
$$

(*) Laboratoire associé au Centre National de la Recherche Scientifique.
Dans ces expressions, $\mathbf{k}$ est l'opérateur impulsion relative des deux nucléons 1 et $2, \mathbf{k}^{\prime}$ son complexe conjugué, $P_{\sigma}$ l'opérateur d'échange de spins et

\begin{tabular}{|c|c|c|c|c|c|c|}
\hline Force & $t_{0}$ & $t_{1}$ & $t_{2}$ & $t_{3}$ & $W$ & $x_{0}$ \\
\hline - & - & - & - & - & - & - \\
\hline I & $-1057,3$ & 235,9 & -100 & 14463,5 & 120 & 0,56 \\
\hline II & $-1169,9$ & 585,6 & $-27,1$ & 9331,1 & 105 & 0,34 \\
\hline III & $-1128,75$ & 395 & -95 & 14000 & 120 & 0,45 \\
\hline IV & $-1205,6$ & 765 & 35 & 5000 & 150 & 0,05 \\
\hline V & 248,29 & 970,56 & 107,22 & 0 & 150 & $-0,17$ \\
\hline VI & $-1101,81$ & 271,667 & $-138,33$ & 17000 & 115 & 0,583 \\
\hline
\end{tabular}

$$
\delta_{i j}=\delta\left(\mathbf{r}_{i}-\mathbf{r}_{j}\right) .
$$

Les quantités $t_{0}, t_{1}, t_{2}, t_{3}, x_{0}$ et $W$ sont des paramètres dont il existe à l'heure actuelle six ensembles. Ils sont donnés dans le tableau suivant

Ce qui est à remarquer dans ce tableau, c'est la différence importante dans les valeurs adoptées pour les paramètres $t_{1}$ et $t_{3}$ d'un ensemble à un autre. Nous reviendrons sur ce point par la suite.

En dehors des trois composantes principales données plus haut qui sont les seules à jouer un rôle dans l'étude des noyaux avec des nombres pairs de protons et neutrons, une composante tenseur a été également introduite pour l'étude des noyaux de masse paire. Elle s'écrit couramment sous la forme

$$
\begin{aligned}
& V_{\mathrm{t}}= T_{0}\left[3\left(\sigma_{1} \cdot \mathbf{k}^{\prime}\right) \delta_{12}\left(\sigma_{2} \cdot \mathbf{k}\right)-\left(\sigma_{1} \cdot \sigma_{2}\right) \mathbf{k}^{\prime} . \delta_{12} \mathbf{k}\right] \\
& T_{\mathrm{E}}\left\{\left[3\left(\sigma_{1} \cdot \mathbf{k}^{\prime}\right)\left(\sigma_{2} \cdot \mathbf{k}^{\prime}\right)-\left(\sigma_{1} \cdot \sigma_{2}\right) \mathbf{k}^{\prime 2}\right] \delta_{12}\right. \\
&\left.\delta_{12}\left[3\left(\sigma_{1} \cdot \mathbf{k}\right)\left(\sigma_{2} \cdot \mathbf{k}\right)-\left(\sigma_{1} \cdot \sigma_{2}\right) \mathbf{k}^{2}\right]\right\} .
\end{aligned}
$$

Les valeurs suivantes ont été trouvées [3] pour les 
paramètres $T_{0}$ et $T_{\mathrm{E}}:\left(T_{0}, T_{\mathrm{E}}\right)=(136,108)$ et $(65,54)$ en $\mathrm{MeV} . \mathrm{fm}^{5}$. D'autre part, la force à trois corps peut être remplacée par une force équivalente à deux corps dépendant de la densité

$$
\bar{V}_{3}=\frac{t_{3}}{6} \rho\left(r_{1}\right) \delta_{12} \text {. }
$$

Ces deux formes donnent exactement le même résultat pour un système ayant la symétrie par renversement du temps.

Dans un système en condensation pionique, les nucléons possèdent une structure en spin-isospin tout à fait particulière. Il a été montré [4] en effet que ce condensat de pions ne peut exister que si la structure de l'état fondamental des nucléons est telle que :

$$
\nabla . \mathbf{S}_{i}(\mathbf{r}) \neq 0
$$

où $\mathbf{S}_{i}(\mathbf{r})$ est la fonction de spin-isospin

$$
\mathbf{S}_{i}(\mathbf{r})=\left\langle\phi_{0}\left|\psi_{i}^{+}(\mathbf{r}) \tau_{3} \boldsymbol{\sigma} \psi_{i}(\mathbf{r})\right| \phi_{0}\right\rangle
$$

$\psi_{i}(\mathbf{r})$ étant le champ de nucléon $i$.

Pour un système infini, un modèle de structure dite de spins alternés (SA) a été conçu, qui permet de tirer au maximum profit de la force tenseur. Il consiste à ranger les nucléons sur des couches parallèles avec la structure en spin-isospin suivante : d'une part, sur chaque couche, les spins des protons ont la même direction qui est opposée à celle des neutrons et, d'autre part, ces spins changent de direction en allant à la couche suivante. La modulation de la distribution de masse dans la direction de l'impulsion des pions condensés que nous prenons comme l'axe des $z$, peut être décrite par les fonctions d'onde à un nucléon

$$
\begin{gathered}
\varphi_{\mathbf{q}_{\perp} l_{v_{l}}}(\mathbf{r})=\Omega_{\perp}^{-1 / 2} \mathrm{e}^{i \mathbf{q}_{\perp} \cdot \rho} \lambda_{l}(z) \chi_{v_{l}} \\
\lambda_{l}(z)=(a / \pi)^{1 / 4} \exp \left(-a(z-l d)^{2} / 2\right) .
\end{gathered}
$$

Dans ces expressions, $d$ est la distance entre deux couches successives et $l$ désigne la couche où est placé le nucléon. D'autre part, $v_{l}=\left(\sigma_{l}, \tau_{l}\right)$ est l'indice de spin-isospin et l'on a supposé que le mouvement dans le plan $\rho=(x, y)$ est un mouvement libre caractérisé par l'impulsion $\mathbf{q}_{\perp}$.

Dans l'étude des systèmes nucléaires usuels possédant la symétrie par renversement du temps, la force à trois corps joue un rôle important pour leur saturation. Considérons maintenant un système de nucléons où cette symétrie est brisée pour lequel la densité de spin-isospin $\mathbf{S}_{i} \neq 0$. Il est facile de montrer que [5]

$$
\begin{aligned}
\left\langle V_{3}\right\rangle=\frac{t_{3}}{4} \int \operatorname{dr}[ & \rho_{\mathbf{p}}^{2}(\mathbf{r}) \rho_{\mathrm{n}}(\mathbf{r})+\rho_{\mathrm{n}}^{2}(\mathbf{r}) \rho_{\mathrm{p}}(\mathbf{r}) \\
& \left.-\mathbf{S}_{\mathbf{p}}^{2}(\mathbf{r}) \rho_{\mathrm{n}}(\mathbf{r})-\mathbf{S}_{\mathbf{n}}^{2}(\mathbf{r}) \rho_{\mathrm{p}}(\mathbf{r})\right] .
\end{aligned}
$$

On voit que la non nullité de $\mathbf{S}_{i}$ entraîne une diminution de l'effet répulsif de la force à trois corps lorsque celle-ci est écrite sous la forme indépendante de la densité.
La structure en spin-isospin du système en condensation pionique décrite plus haut est telle que $\mathbf{S}_{i} \neq 0$. On peut s'attendre à ce que le même effet se produise. Utilisant les deux formes de la force à trois corps, on obtient explicitement

$$
\begin{aligned}
& \left\langle V_{3}\right\rangle=\frac{t_{3}}{6} \frac{\Gamma}{\pi \sqrt{3}}\left[\left(\rho_{\mathrm{p}}+\rho_{\mathrm{n}}\right)^{3} F_{\mathrm{a}}+2\left(\rho_{\mathrm{p}}^{3}+\rho_{\mathrm{n}}^{3}\right) F_{\mathrm{c}}\right. \\
& \left.-3\left(\rho_{\mathrm{p}}+\rho_{\mathrm{n}}\right)\left(\rho_{\mathrm{p}}^{2}+\rho_{\mathrm{n}}^{2}\right) F_{\mathrm{b}}\right] \\
& \left\langle\bar{V}_{3}\right\rangle=\frac{t_{3}}{6} \frac{\Gamma}{2 \pi \sqrt{3}}\left(\rho_{\mathrm{p}}+\rho_{\mathrm{n}}\right)\left[\left(\rho_{\mathrm{p}}+\rho_{\mathrm{n}}\right)^{2} F_{\mathrm{a}}-\right. \\
& \left.-\left(\rho_{\mathrm{p}}^{2}+\rho_{\mathrm{n}}^{2}\right) F_{\mathrm{b}}\right] \\
& F_{\mathrm{a}}=\sum_{m, m^{\prime}} \exp \left\{-\Gamma\left[m^{2}+m^{\prime 2}+\left(m-m^{\prime}\right)^{2}\right] / 3\right\} \\
& F_{\mathrm{b}}=\sum_{m-m^{\prime} \text { pair }} \exp \left\{-\Gamma\left[m^{2}+m^{\prime 2}+\left(m-m^{\prime}\right)^{2}\right] / 3\right\} \\
& F_{\mathrm{c}}=\sum_{m, m^{\prime} \text { pair }} \exp \left\{-\Gamma\left[m^{2}+m^{\prime 2}+\left(m-m^{\prime}\right)^{2}\right] / 3\right\}
\end{aligned}
$$

$\Gamma=a d^{2}$ est un paramètre sans dimension qui caractérise le degré de localisation des nucléons au voisinage des couches. Il est défini par la minimisation de l'énergie totale du système, en même temps que $d$ ou $a$. Dans des cas concrets, on trouve que sa valeur est de l'ordre de 10 , ce qui fait que, avec une bonne approximation

$$
F_{\mathrm{a}} \sim F_{\mathrm{b}} \sim F_{\mathrm{c}} \sim 1 .
$$

D'après les équations (10), (11), on peut donc tirer les conclusions suivantes :

- pour la matière neutronique $\left(\rho_{\mathrm{p}}=0\right)$, la contribution de la force à trois corps est nulle lorsque l'état fondamental possède la structure en spins alternés ;

- pour la matière nucléaire $\left(\rho_{\mathrm{p}}=\rho_{\mathrm{n}}\right)$, seule la forme à deux corps équivalente $\bar{V}_{3}$ donne une contribution non nulle dans un état ayant la même structure.

Par conséquent, la saturation de la force de Skyrme ne peut pas être obtenue principalement grâce à la force à trois corps comme pour le cas des systèmes ayant la symétrie par renversement du temps.

Cela étant, examinons dans quelle condition la force de Skyrme peut être saturée. L'effondrement de la structure en spins alternés peut se produire lorsque $a \rightarrow \infty$ et de deux façons :

- soit avec $\Gamma$ fini et $d \rightarrow 0$,

- soit avec $d$ fini et $\Gamma \rightarrow \infty$.

Lorsque $d \rightarrow 0$ et $\Gamma$ fini, la valeur moyenne du hamiltonien total $K^{\boldsymbol{x}}=T+V_{\mathrm{S}}$ où $T$ est l'énergie cinétique, tend vers la limite

$$
\begin{aligned}
& \lim .\langle H\rangle \rightarrow \frac{\Gamma}{d^{2}} {\left[\frac{\hbar^{2}}{4 m}\left(\rho_{\mathrm{p}}+\rho_{\mathrm{n}}\right)+\right.} \\
&\left.+\sqrt{\frac{\Gamma}{2 \pi}} \rho_{\mathrm{p}} \rho_{\mathrm{n}}\left(\frac{t_{1}}{2}-2 T_{\mathrm{E}}\right) G\right]
\end{aligned}
$$


où

$$
G=\sum_{m \text { pair }} \exp \left(-\Gamma m^{2} / 2\right) \sim 1 .
$$

Il est donc clair que, en faisant tendre la densité vers l'infini, la saturation de la force de Skyrme dépend du signe de la combinaison $t_{1} / 2-2 T_{\mathrm{E}}$. Utilisant les valeurs numériques données plus haut pour $t_{1}$ et $T_{\mathrm{E}}$, on voit que, avec $T_{\mathrm{E}}=65$, il n'y a pas saturation pour les forces $S_{1}$ et $S_{\mathrm{VI}}$ tandis qu'avec $T_{\mathrm{E}}=136$, cette propriété n'est pas satisfaite pour $S_{\mathrm{l}}, S_{\mathrm{III}}$ et $S_{\mathrm{VI}}$. Tout se passe comme si, dans le choix des paramètres pour ces forces, l'accent avait été mis trop lourdement sur la composante à trois corps, au détriment de la composante $t_{1}$. Or la composante à trois corps est fortement réduite pour les systèmes ayant la structure en spins alternés, d'où l'insuffisance de ces forces quant à leur saturation. Le même phénomène a déjà été observé [5] dans l'étude du noyau de ${ }^{14} \mathrm{~N}$ qui possède deux nucléons célibataires (donc ne peut être saturé en spins) et pour lequel un calcul de Hartree-Fock conduit au résultat que l'énergie tend vers $-\infty$ lorsque le rayon du noyau tend vers zéro.

Remarquons que la force de Skyrme a une portée nulle. Pour maintenir cette forme et en même temps obtenir saturation, une plus forte dépendance en densité semble donc être nécesssaire [6]. Bien entendu, il est toujours possible de satisfaire à la condition de saturation en prenant une force à portée finie avec une forte répulsion à courte distance mais cela enlèverait toute la simplicité de la force de Skyrme.

\section{Bibliographie}

[1] SKyrme, T. H. R., Philos. Mag. 1 (1956) 1043.

[2] Beiner, M., Flocard, H., Nguyen van Giai et Quentin, P., Nucl. Phys. A 238 (1975) 29.

[3] Stancu, I., Brink, D. M. et Flocard, H., Phys. Lett. 68B (1977) 108.
[4] Takatsuka, T., Tamya, K., Tatsumi, T. et Tagamaki, R., Prog. Theor. Phys. 59 (1978) 1933.

[5] Chang, B. D., Phys. Lett. 56B (1975) 205.

[6] Backman, S. O., Jackson, A. D. et SPeth, J., Phys. Lett. 56B (1975) 209. 